\section{Prof. Vogl zum Geburtstag}

Am 17. Mai 2018 wird Prof. Dr. Thomas J. Vogl, Direktor des Instituts für Diagnostische und Interventionelle Radiologie des Universitätsklinikums Frankfurt am Main, 60 Jahre alt.

Prof. Vogl wurde in München geboren und studierte nach dem Abitur von 1976 bis 1982 Humanmedizin an der Ludwig-Maximilians-Universität München, an der er auch 1983 promovierte. Anschließend arbeitete er zunächst als wissenschaftlicher Mitarbeiter an der Radiologischen Klinik im Klinikum Großhadern (Direktor: Prof. Dr. Lissner). Im Jahr 1988 erfolgte ein Forschungsaufenthalt am MassachusettsGeneral-Hospital Boston (Leitung: Prof. Dr. Juan Taveras). Im Jahr 1989 erhielt er die Anerkennung als Arzt für Radiologie mit Fachkunde Strahlentherapie, im Jahr 1992 die Anerkennung für Nuklearmedizin und in den Jahren 1992 und 1996 die für Neuroradiologie. Im Jahr 1993 folgte die Habilitation und er nahm den Ruf für eine C3-Professur für allgemeine Radiologie an der Charité Berlin an und wurde zum leitenden Oberarzt am Universitätsklinikum Rudolf Virchow ernannt (Direktor: Prof. Dr. Dr. h. c. R. Felix).

Der klinische und wissenschaftliche Schwerpunkt von Prof. Vogl liegt in der Diagnostik und interventionellen Therapie tumoröser Erkrankungen. Insbesondere in der lokalen Therapie hepatischer Neoplasien hat er sich einen internationalen Ruf aufgebaut. Hier ist die von ihm patentierte laserinduzierte Thermotherapie (LITT) zu erwähnen. Durch seinen Einsatz konnte insbesondere die thermische Ablation von hepatozellulären Karzinomen und Lebermetastasen als kurative Option bei inoperablen Befunden etabliert werden. Hierfür wurde ihm 1994 der Marie-Curie-Ring der Deutschen Röntgengesellschaft verliehen. Seine Erfahrungen konnte er in neue Ablationsverfahren und auf unterschiedliche Tumorlokalisationen einbringen, sodass diese Verfahren auch heute noch einer großen Patientengruppe als alternative, schonende und minimalinvasive Therapie angeboten werden können.
Im Jahr 1998 folgte er einem C4-Ruf als Professor für allgemeine Röntgendiagnostik an die Frankfurter Goethe-Universität. Dort konnte er mit seinem Team die Abteilung prägen und seinen Schwerpunkt in den neuen Abteilungsnamen einbringen: „Institut für Diagnostische und Interventionelle Radiologie“.

Darüber hinaus ist die Bildgebung der Kopf-Hals-Region, und insbesondere die Detektion von HNO-Tumoren, ein wichtiger Fokus von Prof. Vogl sowie des Instituts. Ein weiterer Schwerpunkt seiner Tätigkeit ist die Verfeinerung diagnostischer Möglichkeiten mit den modernsten Geräten der Schnittbildverfahren. Neben interventionellen Biopsieverfahren im CT und MRT werden auch funktionelle Darstellungen erprobt und weiterentwickelt - wie die dynamische Bildgebung des Kiefergelenks oder des schlagenden Herzens.

Prof. Vogl hat die Radiologie stets als interdisziplinäres Fach verstanden. Daraus resultiert auch der enge Kontakt mit den klinischen Partnern. Dies zeigt sich in der Fülle der gemeinsamen Bilddemonstrationen, von denen z. B. die Allgemeinchirurgie und HNO-Bildgebung bis heute persönlich von ihm übernommen werden.

Seine Tätigkeit als stellvertretender ärztlicher Direktor des Klinikums der JohannWolfgang-Goethe-Universität Frankfurt in den Jahren 2005 bis 2011 gab ihm die Möglichkeit, unterschiedliche Kooperationen zu planen und interdisziplinäre Projekte zu verwirklichen.

So konnten enge Kooperationen mit den Zentren der Inneren Medizin, der Chirurgie, HNO, Dermatologie, Augenheilkunde, Rechtsmedizin und auswärtigen Krankenhäusern eingegangen bzw. ausgebaut werden, die zu einer Vielzahl von wissenschaftlichen Beiträgen und Veröffentlichungen geführt haben und letztendlich im Zeitraum von 20 Jahren insgesamt 21 Mitarbeitern die Möglichkeit zu einer Habilitation gegeben haben.

Ein wichtiges Anliegen war es für Prof. Vogl auch immer, die Radiologie als klinisches Fach in der Öffentlichkeit darzustellen. Er agiert regelmäßig als Interviewpartner für Radio- oder Fernsehsender, zuletzt zum

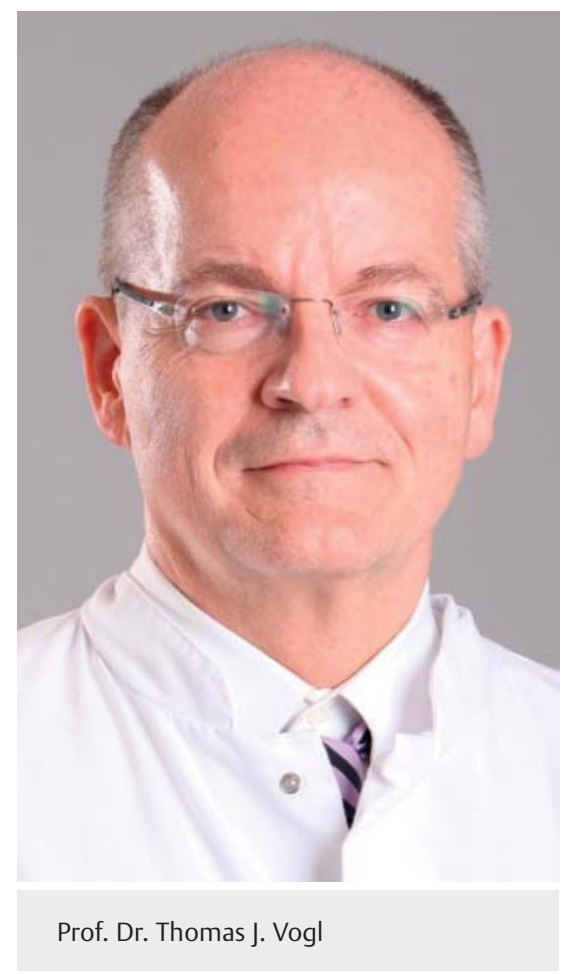

Thema „Gadoliniumablagerungen im Gehirn“. Häufig finden sich am Institut Kamerateams von Fernsehsendern ein, welche über neue Therapieverfahren berichten oder Patienten bei einer Behandlung begleiten.

Die Atmosphäre an seinem Institut ist geprägt durch einen kollegialen, stets vermittelnden Arbeitsstil. Die Begeisterung für das Fach ist ihm jeden Tag aufs Neue anzumerken - selbst weit vor 6 Uhr morgens zu seinem Arbeitsbeginn. Zudem ist hier auch seine außerordentliche Förderung von jungen Kollegen zu nennen, die er stets für die Forschung motivieren kann und denen er auch früh Verantwortung übergibt. Er sucht und findet in jedem Mitarbeiter „das Leuchten in den Augen“ für das Fach Radiologie.

Wir wünschen ihm von Herzen alles Gute zu seinem Geburtstag!

Dr. med. Julian L. Wichmann, Frankfurt/ Main

Prof. Dr. med. Katrin Eichler, Frankfurt/Main Prof. Dott. Univ. Pisa med. Volkmar Jacobi, Frankfurt/Main 